\title{
Terahertz Sub-Nanometer Sub-Surface Imaging of 2D Materials
}

\section{Rahman $A^{1 *}$, Rahman AK $^{1}$, Yamamoto $\mathrm{T}^{2}$ and Kitagawa $\mathrm{H}^{2}$}

${ }^{1}$ Applied Research and Photonics, Friendship Road, Harrisburg, United States

${ }^{2}$ Graduate School of Science, Kyoto University, Kyoto-fu, Japan

\begin{abstract}
Terahertz sub-surface imaging offers an effective solution for surface and 3D imaging because of minimal sample preparation requirements and its ability to "see" below the surface. Another important property is the ability to inspect on a layer-by layer basis via a non-contact route, non-destructive route. Terahertz 3D imager designed at Applied Research and Photonics (Harrisburg, PA) has been used to demonstrate reconstructive imaging with a resolution of less than a nanometer. Gridding with inverse distance to power equations has been described for 3D image formation. A continuous wave terahertz source derived from dendrimer dipole excitation has been used for reflection mode scanning in the three orthogonal directions. Both $2 \mathrm{D}$ and $3 \mathrm{D}$ images are generated for the analysis of silver iodide quantum dots' size parameter. Layer by layer image analysis has been outlined. Graphical analysis was used for particle size and layer thickness determinations. The demonstrated results of quantum dot particle size checks well with those determined by TEM micrograph and powder X-ray diffraction analysis. The reported non-contact measurement system is expected to be useful for characterizing $2 \mathrm{D}$ and $3 \mathrm{D}$ naomaterials as well as for process development and/or quality inspection at the production line.
\end{abstract}

Keywords: Terahertz 3D sub-surface imaging; Non-contact and non-destructive scanning; Layer by layer inspection; Nanoparticle size determination; Quantum dot imaging

\section{Introduction}

Inspection of smaller dimensions for $2 \mathrm{D}$ and $3 \mathrm{D}$ nanomaterials and interfaces is a critical need for the next generation of technologies featuring $<10 \mathrm{~nm}$ patterns and 3D ICs. It is also important to be able to inspect the under-layer features via a non-destructive technique for both the process development and post-process phases of an IC development. Nanometer inspection metrology is, thus, critical for the yield enhancement and process monitoring phases of semiconductor manufacturing. In this paper we describe a new technique of $3 \mathrm{D}$ imaging with layer-by-layer inspection capability in a non-contact fashion with a resolution approaching less than nanometer.

Sub-nanometer imaging resolution and determination of nanoparticles' size distribution capabilities were achieved via terahertz techniques, where, a high power, continuous wave (CW) terahertz energy (T-ray) was utilized for interrogation. Here, T-ray was generated via dendrimer dipole excitation (DDE) mechanism [1]. The traditional optical route for high resolution imaging is limited by the fact that it cannot resolve feature sizes smaller than the wavelength of the light used in measurements. While X-rays have wavelength of $\sim 10 \mathrm{~nm}$ or less, it is not feasible for delicate inspections because of its ionizing nature. Modern AFMs can reach lateral resolution of $\sim 30 \mathrm{~nm}$; however, this is strictly a surface topography technique for small samples; not feasible for sub-surface inspection, or for a large samples such as a semiconductor wafer. Therefore, a capability of non-destructive, non-contact, sub-surface imaging; with a resolution of $\sim 1 \mathrm{~nm}$ in all 3 dimensions with adjustable stand-off distance, is important. Although terahertz wavelength is bigger than infrared and other waves mentioned above; however, we exploit its penetration ability in conjunction with a reconstructive imaging system for capturing response from small features in the nano-scale range via an algorithm called gridding with inverse distance to power equations [2-4].

T-ray belongs to a narrow band of energy lying between infrared and microwave frequencies. It was shown in the late $80 \mathrm{~s}$ that T-ray can be generated using a femto-second pulsed laser (femto-laser) that came to be known as a photoconductive antenna or the Auston switch [5]. This technique is dependent on a femto-laser that is bulky and costly. Later, Robert F Curl (NL'89) group demonstrated that farinfrared (terahertz) waves may be generated by diode lasers without requiring a femto-laser [6]. However, it was still a low power source without much practical application. Previously, we reported that an organic nanomaterial called "dendrimer" possesses properties that can be exploited for higher electro-optic response by engineering a higher dipole population. This electro-optic dendrimer was used for high power, $\mathrm{CW}$, wide-band terahertz generation via a mechanism called dendrimer dipole excitation or "DDE" [1]. This new source provides improved efficiency, with an output power of a few 10s of milliwatts in the CW mode. This development in terahertz generation enables a non-destructive testing of 2D materials, interfaces and other substrates on a layer by layer basis.

The principle of semiconductor defect detection was described elsewhere [2]. Briefly, the intensity of reflected terahertz beam is measured and modeled via a modified Beer-Lambert's law. For a given pattern on a wafer, all material parameters remain unchanged during measurements, because, terahertz radiation is non-ionizing and does not perturb the intrinsic properties. Thus, the reflectance is proportional to the given material at the point where the beam is incident. As such, the reflectance is dependent on the spatial and angular coordinates. The Beer-Lambert's law may be restated in terms of the reflectance and a physical parameter:

$R(r)=\varepsilon(r) . l(r) . \rho(r)$, where the reflectance is coordinate dependent,

*Corresponding author: Anis Rahman, Applied Research and Photonics, 470 Friendship Road, Suite 10, Harrisburg, PA 17111, United States, Tel: +1-717-6238201; E-mail: a.rahman@arphotonics.net

Received September 14, 2016; Accepted September 22, 2016; Published September 30, 2016

Citation: Rahman A, Rahman AK, Yamamoto T, Kitagawa H (2016) Terahertz Sub-Nanometer Sub-Surface Imaging of 2D Materials. J Biosens Bioelectron 7: 221. doi: $10.4172 / 2155-6210.1000221$

Copyright: (c) 2016 Rahman A, et al. This is an open-access article distributed under the terms of the Creative Commons Attribution License, which permits unrestricted use, distribution, and reproduction in any medium, provided the original author and source are credited. 
which also causes variation in the path length, $l(r)$, and consequently, variation in the coefficient, $\rho(r)$. A 3D reconstructed image generated from the measured reflectance, therefore, will yield the characteristic features (patterns). Since T-Ray can penetrate silicon and other semiconductors, the energy may be reflected off of a given interface by a suitable focusing arrangement. So, if there is a void or other defect in any of the sub-surface layers, that will be identifiable from both reflected and transmitted intensities; as well as from transreflectance responses.

In what follows, we first describe the characterization of terahertz beam that is delivered by a fiber-optic probe. We then outline some details of the reconstructive imaging process that is followed by a calibration of the naoscanner. Afterwards, we describe a practical example of reconstructive imaging of silver iodide (AgI) quantum dots along with measurements of nanoparticle size via graphical analysis. A few concluding remarks are given at the end.

\section{Terahertz beam characterization}

A fiber coupled terahertz time-domain spectrometer and nanoscanner has been designed around the aforementioned DDE terahertz source [1]. The generated terahertz beam was coupled in to a multimode fiber for delivery to the sample. First a CW pump laser is split in to two arms of 80:20 ratio, similar to a spectrometer described elsewhere [5]. The 80 -arm, called pump-arm, is used to excite the aligned dipole population in the electro-optic dendrimer [1]. The 20 -arm is called the probing arm that is combined with the pump-arm before coupling in to the fiber. An NEC terahertz camera (NEC IRV-T0831) is used for characterizing the beam (Figure 1) at the delivery point before passing through the focusing optics and being incident on the sample (Figure 1).

Figure 2a shows the intensity distribution of the beam out of the multimode fiber, as seen by the camera in Figure 1 where the beam diameter is approximately $3 \mathrm{~mm}$. A graphical analysis along the line is shown in Figure 2b; where the half width at full maximum (FWFM) is $\sim 1.5 \mathrm{~mm}$. This beam is fed in to the focusing optics for interrogating the samples. The beam finally passes through a tunable aperture before being incident on the sample. While the beam appears to be roughly a top-hat shape at the end of the fiber, in reality it is expected to be of Gaussian shape when incident on a sample; thus the incident tip of the beam is smaller than that shown in Figure 2. Since all reflection is a diffused reflection from a real sample, the reflected beam is also of Gaussian shape. On the receiving end where the beam enters the detection system, a combination of a microscope objective and a dendrimer filter is used that truncates the diffused part of the beam; only a very small tip corresponding to the reflecting object is captured. This ensures a sharp image formation on the nanometer scale (Figure 2).

\section{Reconstructive imaging leading to nanometer resolution}

The principle of reconstructive imaging is well understood; however, what is challenging is to achieve higher resolution, as explained before. All direct imaging methods involve either a charged coupled device or other kind of sensing array; output of which is converted in to an image. As such the pixel size is defined by the size of the array elements of the sensor/CCD used for image generation. Reconstructive imaging offers an important opportunity to define one's own pixel size (or voxel size) by a hardware and software combination. The procedure is outlined below.

Consider Figure 3 where the trace of a semiconductor test wafer with different patterns is shown. The trace demonstrates that distinct materials may be identified based on their reflectance. However, this also forms the basis of reconstructive imaging. If different materials are placed close by, as in the case of a semiconductor wafer; and if a scan is performed to capture the intensity reflected by each feature then the reflected intensity may be processed to form an image that will represent the given feature; just like a CCD forms an image based on the incident light on it. Unlike a CCD, where the pixel sized is defined by its array elements, however, here the pixel size is defined by the smallest displacement of the scanning mechanism that captures the intensity and the algorithm that generates the image (Figure 3).

Data structure: 3D imaging requires a value of a voxel, which is the smallest unit corresponding to a $3 \mathrm{D}$ space; i.e., $\{x, y, z, v\}$, where $\{x, y, z\}$ are the three orthogonal coordinates and is the data at that point. To characterize a $3 \mathrm{D}$ space, data need to be generated for all over a given $3 \mathrm{D}$ volume. This is best done by an experimental scanning protocol where the volume is divided in to a number of slices (surfaces) and the slices are scanned one after another. Thus, the data are generated in the following sequence: for every $\left\{z_{1}, y_{1}\right\}$, a line is scanned giving $\left\{x_{1}, x_{2} \ldots\right.$ $\left.x_{n}\right\}$. Then the line is repeated for $y_{2}$ through $y_{n}$, while keeping the $z_{1}$ (i.e., the depth) fixed. This sequence of line at a given interval generated the first slice of the volume. Then the whole scan is repeated for $z_{2}$, yielding the second slice of the volume. This process is then repeated for all the slices to scan the whole volume. The line scan is done by a streaming data acquisition algorithm, where, a command is issued to move the scanner from the start point to the end point along the $x$-axis. As the positioning stage moves, its instantaneous position is recorded by the computer interface; thus generating the $\left\{x_{1}, \ldots x_{n}\right\}$ points for a given $\left\{z_{i}, y_{j}\right\}$. The reflected intensity or both the reflected and transmitted intensity is read simultaneously corresponding to each. Once the whole volume is scanned, the data set is then used for generating the image via inverse distance to power equations.

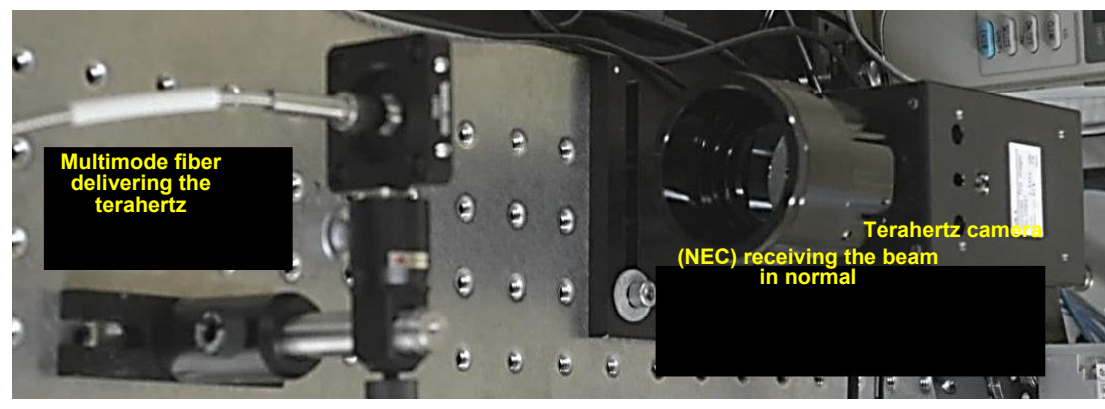

Figure 1: Beam characterization setup. Multimode fiber head is delivering the beam in to the terahertz camera (NEC IRV-T0831). Fiber to camera distance is $\sim 5 "$. 


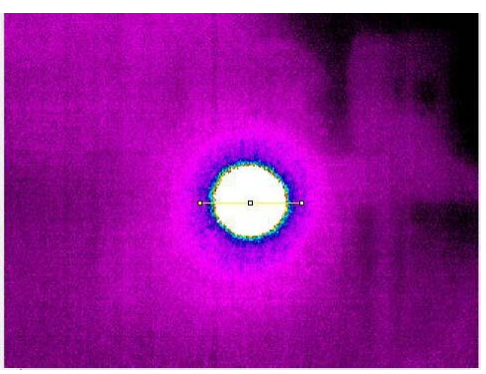

(a)

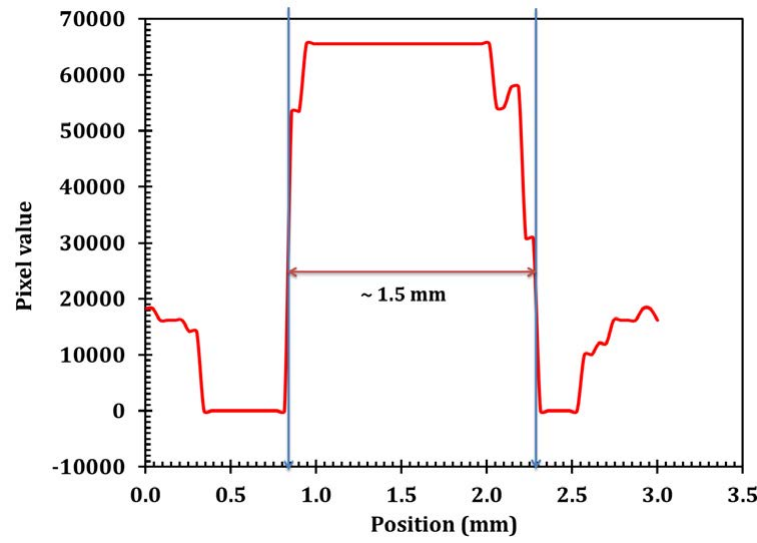

(b)

Figure 2: (a) Beam spot as detected by the terahertz camera. (b) Analysis along the line ( $3 \mathrm{~mm}$ ) reveals a top hat shape at a distance of $\sim 5$ " with a FWHM $\sim 1.5 \mathrm{~mm}$.

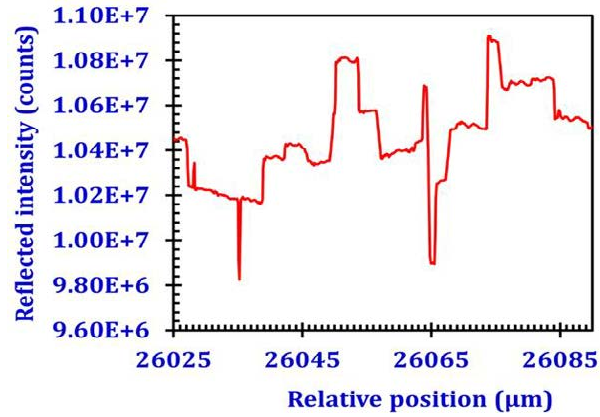

Figure 3: Reflected intensity is proportional to material property; therefore, change in intensity indicates a difference in material property. This forms the basis for nanometer resolution imaging.

Inverse distance to power equations: This is a method for gridbased map creation from measured $\{x$, $\}$ data set. Practical $\{x$, based data typically comprise of irregularly spaced values; as such requires further computation to generate a grid-based map. The gridding process effectively interpolates data values for the lattice at locations where data values are absent. Therefore, closer the measured data points to each other, more accurate is the gridded image for feature sizes that are smaller than the hardware resolution. The current experimental setup has a hardware resolution of $<25 \mathrm{~nm}$ (Figure 4). Therefore, the interpolation via inverse gridding method may be used to generate an image at $1 \mathrm{~nm}$ resolution or less. The reliability of the interpolation is tested by calibration with respect to known dimensions (Figure 4).

A smoothing parameter may be applied during interpolation in order to suit the imaging requirements for a given specimen. The method does not extrapolate values beyond those found in the source data. The following equations are used for computation of the $3 \mathrm{D}$ lattice via inverse distance to a power $[3,4]$ :

$$
\hat{C} \boldsymbol{j}=\frac{\sum_{i}^{n}=\mathbf{1} \frac{c_{i}}{h_{i j}^{\hat{\alpha}}}}{\sum_{i}^{n}=\mathbf{1} \frac{1}{h_{i j}^{\hat{a}}}},
$$

Where, $\boldsymbol{h}_{i j}=\sqrt{d_{i j}^{2}+\delta^{2}}$
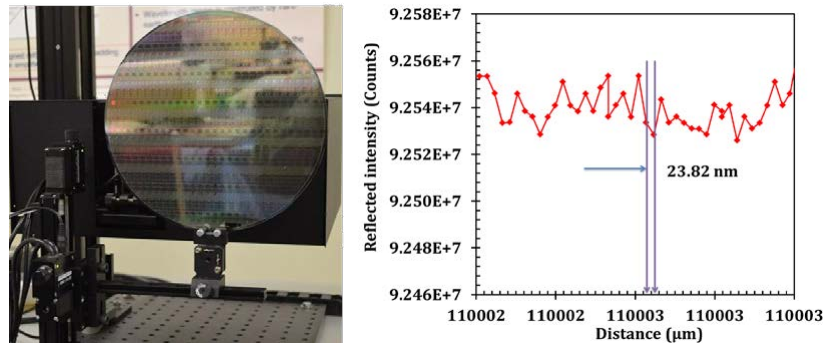

Figure 4: (a) A test wafer is mounted on the nanoscanner; (b) lateral hardware resolution of scanner is $\sim 24 \mathrm{~nm}$.

$\mathrm{h}_{i j}$ is the effective separation distance between grid node " $j$ " and the neighboring point " $i$ ";

$\widehat{C} j$ are the interpolated values for lattice node " $j$ ";

$C_{i}$ are the neighboring measured points;

$d i j$ is the distance between grid node " $j$ " and the neighboring point " $i$ ";

$\beta$ is the power or weighting parameter; and

$\delta$ is the smoothing parameter.

The power, $\beta$ and the smoothing factor, $\delta$, in the above computation 
may be chosen by the user to suit different imaging needs. Once the lattice is calculated, the surface image and volume image is generated by simply rendering the grid with a chosen color scheme. As an illustration of this technique, consider a function, $f(x, y, z)=C * \sin (x)$ to demonstrate the image formation. One can easily compute this function over a given $3 \mathrm{D}$ space. Let us assume: $x \rightarrow 0 \ldots 2 \pi, y \rightarrow 0 \ldots 6$, $z \rightarrow 0 \ldots 6$. Once the function is evaluated via the procedure described above, one can construct the data space. Then using the gridding method, one can reconstruct (map) the function over the given 3D space. The plot for the above function looks like as shown in Figure

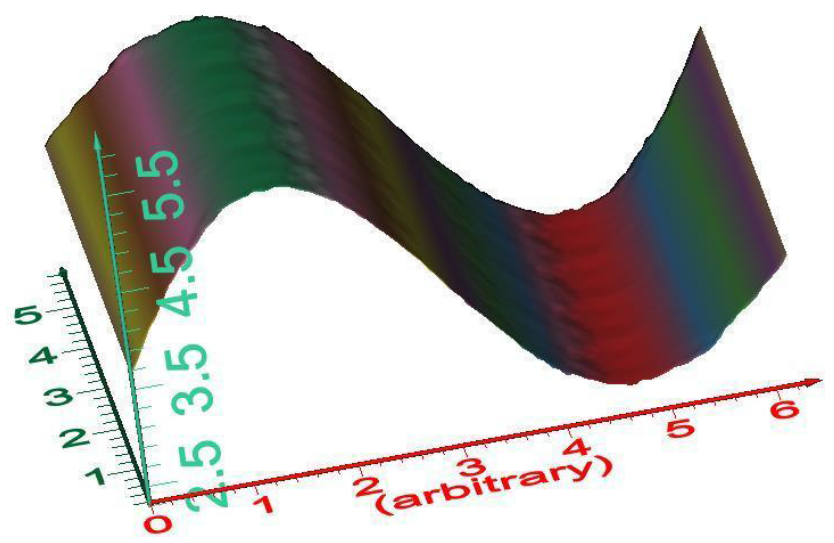

Figure 5: 3D plot of the function $f(x, y, z)=c{ }^{*} \sin (x)$.
5. Closer the grid points, smoother will be the surface. One can plot experimental data by the same procedure (Figure 5).

\section{Calibration}

A photograph of the terahertz nanoscanner is shown in Figure 3a. A semiconductor test wafer is placed on a 3 axis $(\mathrm{X}, \mathrm{Y}$, and $\mathrm{Z})$ positioning stage in front of the $\mathrm{THz}$ source and detection system. The positioners have the maximum speed of $\sim 7 \mathrm{~mm} / \mathrm{s}$ and the minimum speed of 500 $\mathrm{nm} / \mathrm{s}$ with the highest lateral resolution $\sim 24 \mathrm{~nm}$ (Figure $3 \mathrm{~b}$ ) achieved via a data streaming algorithm. A digital video microscope was used to pick out different structures with different sizes on the test wafer to measure the actual size of these features. A few of the features were identified on the test wafer and marked for scan alignment. Each scan recorded the changes in reflected intensity based on changes in the position, and therefore, the pattern on the wafer; all data were stored in a file for subsequent analysis.

A picture of the chosen spot was recorded by a digital microscope. Figure 6 shows the reproducibility of the scanned traces and Figure 7 shows an optical micrograph of a segment of the wafer against the microscope standard. The features on the wafer were marked as \#1 through \#8 and shown in Table 1 . The dimensions of these features were determined via the microscope reading. The same features were then scanned on the nanoscanner. To insure that the results of the small features are accurate, the experiment is repeated using both larger and smaller features. The results for the small features are shown in Table 1. An average calibration factor of 0.998 was established. In the future, even smaller lithography features may be used for calibration as they

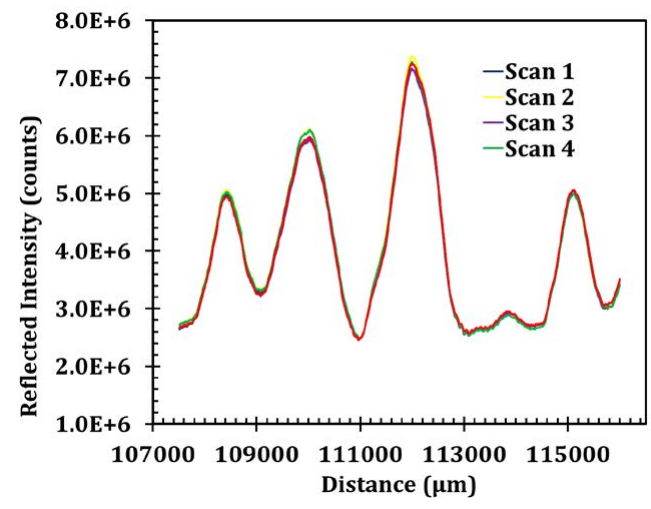

Figure 6: Reproducibility of scan for identified features on a test wafer.

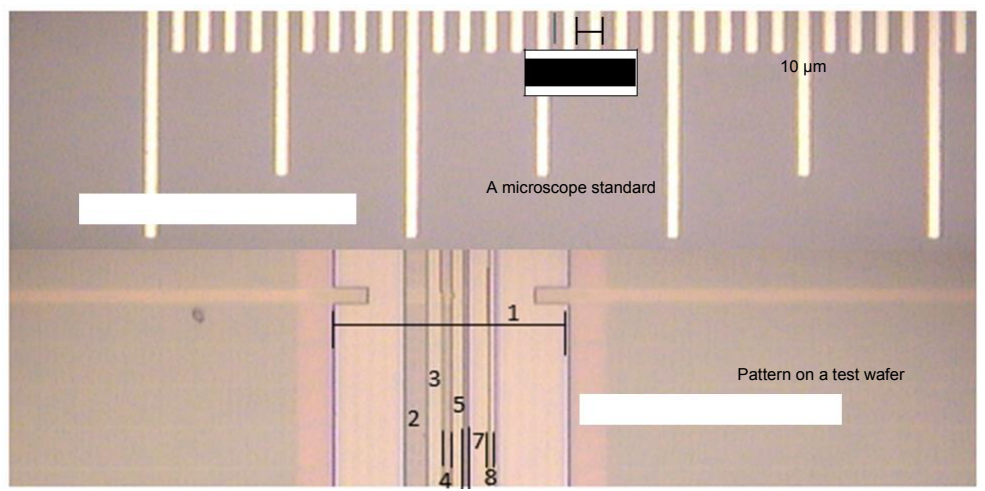

Figure 7: Optical image used to calibrate the scanned features. Top: a photomicrograph of a microscope standard; and bottom: features on the test wafer (see Figure 3(a)). Each number shows a pattern with a different dimension. Both the top and bottom images are under identical magnification. 
Citation: Rahman A, Rahman AK, Yamamoto T, Kitagawa H (2016) Terahertz Sub-Nanometer Sub-Surface Imaging of 2D Materials. J Biosens Bioelectron 7: 221. doi: 10.4172/2155-6210.1000221

Page 5 of 8

\begin{tabular}{|c|c|c|c|c|}
\hline $\begin{array}{c}\text { X-scan } \\
\text { (see Figure 4) }\end{array}$ & $\begin{array}{c}\text { Feature size from microscope } \\
\text { calibration }(\mu \mathrm{m})\end{array}$ & $\begin{array}{l}\text { Feature size from the } \\
\text { nanoscanner }(\mu \mathrm{m})\end{array}$ & $\begin{array}{c}\text { Ratio of microscope reading to } \\
\text { nanoscanner reading }\end{array}$ & $\begin{array}{c}\text { Average calibration } \\
\text { Factor }\end{array}$ \\
\hline \#1 & 89.4 & 84.8437 & 0.92666331 & \multirow{8}{*}{0.998} \\
\hline \#2 & 9.6115 & 9.5488 & 0.99347656 & \\
\hline \#3 & 5.3774 & 5.8341 & 1.08492952 & \\
\hline$\# 4$ & 3.9867 & 3.6671 & 0.91983345 & \\
\hline \#5 & 3.9867 & 3.8815 & 0.97361226 & \\
\hline$\# 6$ & 2.4917 & 2.3574 & 0.94610106 & \\
\hline$\# 7$ & 6.8346 & 7.4533 & 1.09052947 & \\
\hline \#8 & 3.3671 & 3.4766 & 1.03252057 & \\
\hline
\end{tabular}

Table 1: Calibration with respect to optical microscope standard.
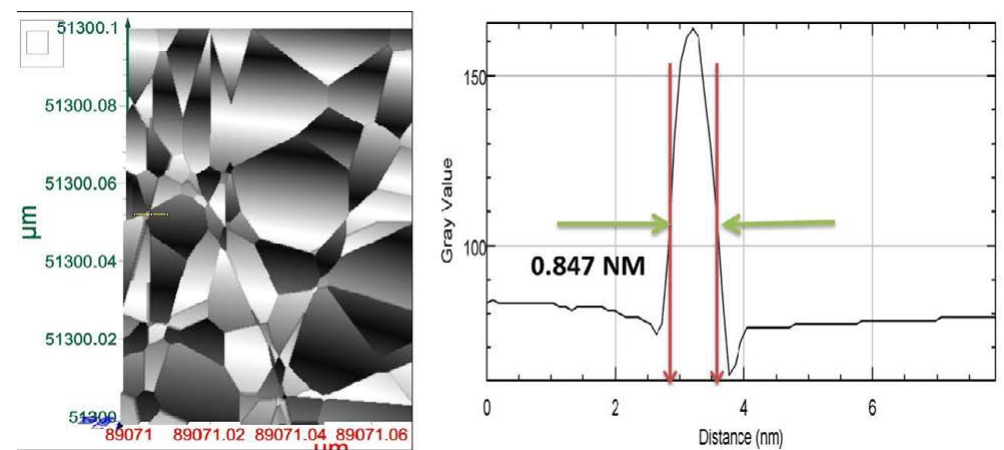

Figure 8: Left: Image of $\mathrm{Cr}^{3}$ nanoparticles on glass slide. Right: Smallest particle detected is $\sim 8.5 \AA(<1 \mathrm{~nm})$

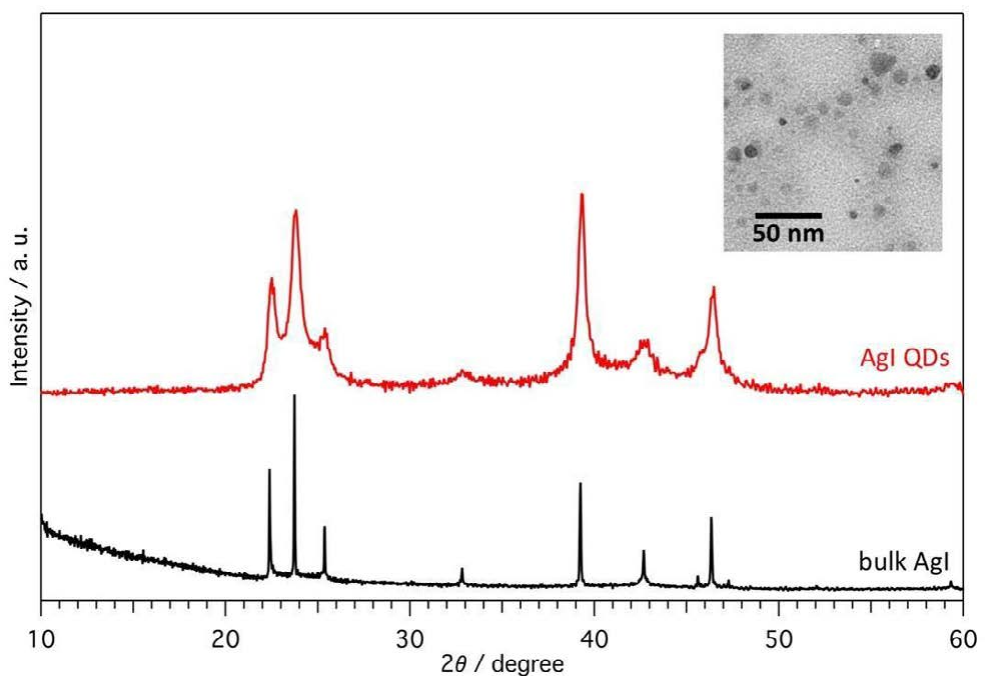

Figure 9: PXRD and TEM analysis shows the Agl quantum dots are $\sim 11 \mathrm{~nm}$.

become available. A resolution of less than $1 \mathrm{~nm}$ was demonstrated from the above described imaging system [7]; as shown in Figure 8. Here, a dilute solution of chromium salt $\left.\left(\mathrm{CH}_{3} \mathrm{CO}_{2}\right)_{7} \mathrm{Cr}_{3}(\mathrm{OH})_{2}\right]$ was spun on a glass slide and a small volume was imaged by the above mentioned method. A slice of the volume was extracted, as shown in Figure 8. Grains and grain boundaries of chromium salt is visible; the smallest particle along the small line has a diameter $\sim 0.85 \mathrm{~nm}$ (Figures $6-8$ and Table 1).

\section{Experimental}

In this section we describe examples of measurements of a practical system. In particular, a sample of silver iodide (AgI) quantum dots (QDs) was imaged and characterized for size parameter.

\section{AgI Quantum Dots 3D analysis and size determination}

AgI quantum dots were synthesized at Kyoto University (Japan) via the procedure reported previously [8]. Briefly, $\mathrm{AgNO}_{3}(1.20 \mathrm{mmol})$ and poly ( $N$-vinyl-2-pyrrolidone $)(2.40 \mathrm{mmol})$ were dissolved in $950 \mathrm{~mL}$ of pure water, and the solution was stirred for 20 minutes at room temperature. Then, $\mathrm{NaI}(4.80 \mathrm{mmol})$ dissolved in $50 \mathrm{~mL}$ of pure water was added quickly and stirred for $2 \mathrm{~h}$. The synthesized quantum dots were collected by filtration, washed with pure water, and dried under vacuum. Analyses via powder X-ray diffraction (XRD) and transmission electron microscope (TEM) (Figure 8) show that the synthesized sample is AgI QDs; where the QDs have a mean diameter of $11.4 \pm 4.5 \mathrm{~nm}$. Terahertz characterization was carried out at Applied 
Citation: Rahman A, Rahman AK, Yamamoto T, Kitagawa H (2016) Terahertz Sub-Nanometer Sub-Surface Imaging of 2D Materials. J Biosens Bioelectron 7: 221. doi: 10.4172/2155-6210.1000221

Page 6 of 8

Research and Photonics, USA. As received sample was dispersed in reagent grade methanol (Sigma Aldrich) at a concentration of $\sim 7.5$ $\mathrm{mg} / \mathrm{mL}$. The solution was spun on a polished silicon wafer at a casting speed of $2000 \mathrm{rpm}$. The resulting film was dried at room temperature and the wafer (containing the film) was mounted on the terahertz nanoscanner, similar to Figure 3a. Five cubic micron $\left(5 \mu \mathrm{m}^{3}\right)$ volume was scanned on a layer by layer basis in the $\mathrm{X}, \mathrm{Y}$, and $\mathrm{Z}$ directions, as described before. Reconstructive imaging and image analysis was conducted as described below [9]. Here, it was assumed that the terahertz interaction with the AgI QDs does not excite these particles for their emission responses. Therefore, no further consideration was given for their emission properties (Figure 9).

\section{Results and Discussion}

Figure 10 shows a surface image of $5 \times 5^{2}$ and also a close-up of $2 \times 2^{2}$

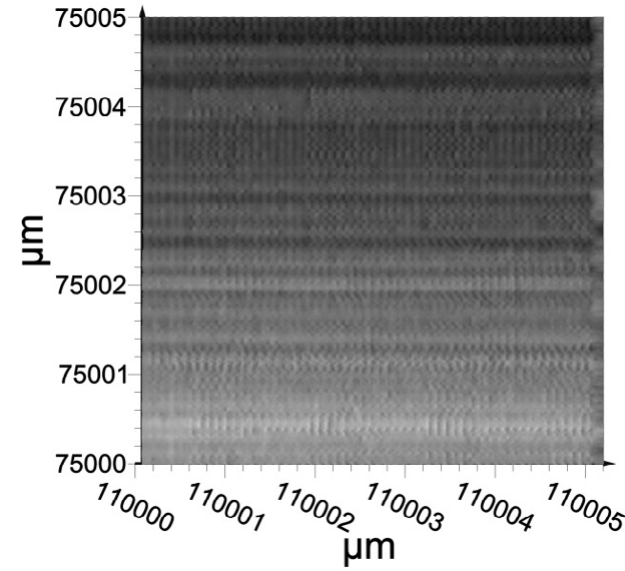

Figure 10: Left: $5 \times 5 \mu \mathrm{m}^{2}$ and right: $2 \times 2 \mu \mathrm{m}^{2}$ surface slices shows random particles distribution.

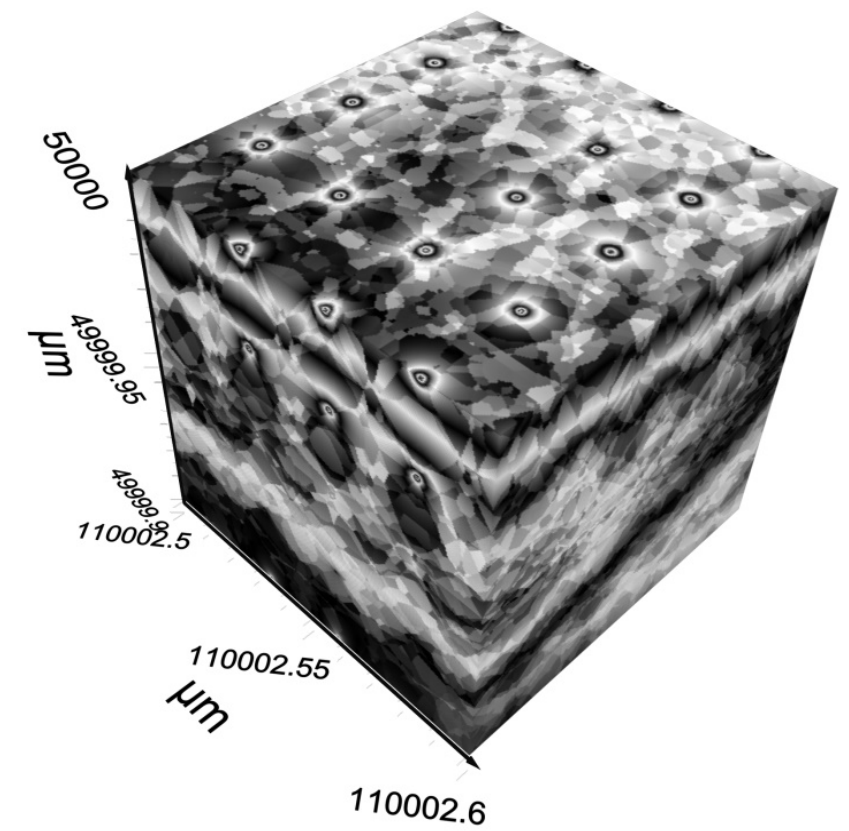

Figure 11: $100 \mathrm{~nm}^{3}$ volume (close up) extracted from the $5 \mu \mathrm{m}^{3}$ scanned volume. Layering structure is visible with the QDs on the top layers. area. A one hundred cubic nanometer $\left(100^{3}\right)$ segment was extracted from the top, as shown in Figure 11; a few individual QD particles formation is visible on the top while a few QD particles are also visible below the top layer. However, there are no other particles visible along the thickness of the $100 \mathrm{~nm}^{3}$ volume. This indicates that most of the QD particles remained on the top of the silicon substrate [10].

Figure 12 shows the top surface of the volume in Figure 11, where, several QD particles are visible; the rest of the pattern on the surface is presumably due to the grains/grain boundaries of silicon oxide on the wafer. A graphical analysis of Figure 12 is presented in Figure 13 which exhibits the distribution of the grains and the QDs along the horizontal line.

Figure 14(a) shows a close-up of the image in Figure 12 that is used to determine the size of a single QD. As shown in the plot if

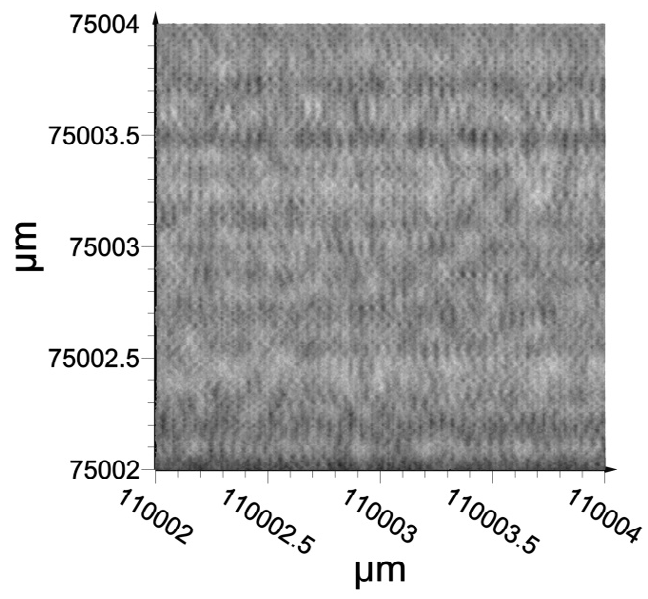




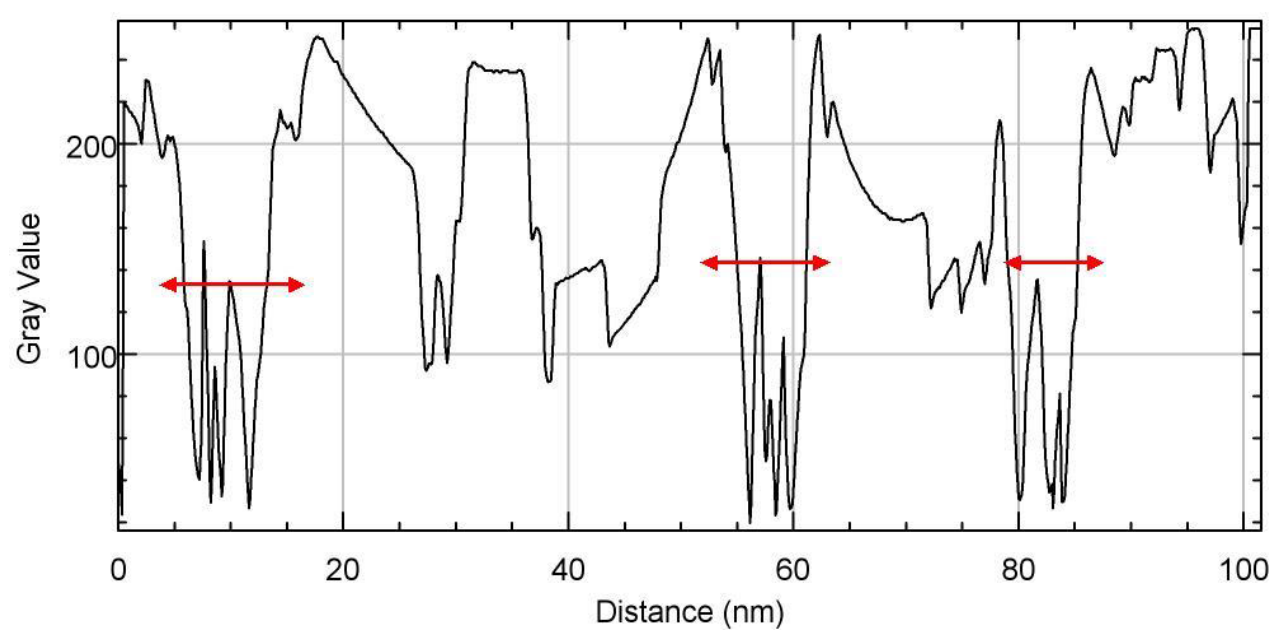

Figure 13: Graphical analysis along the yellow line of Figure 11 showing the size distribution of grains and particles. Particle size is indicated by the arrows.

(a)

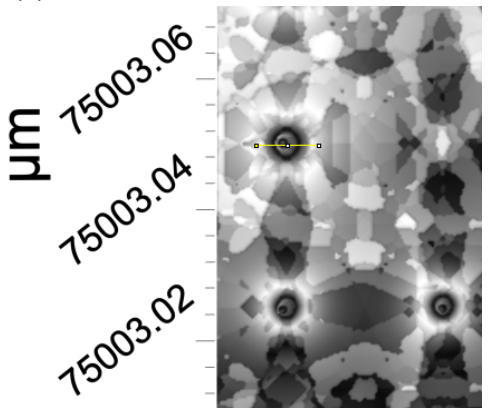

(b)

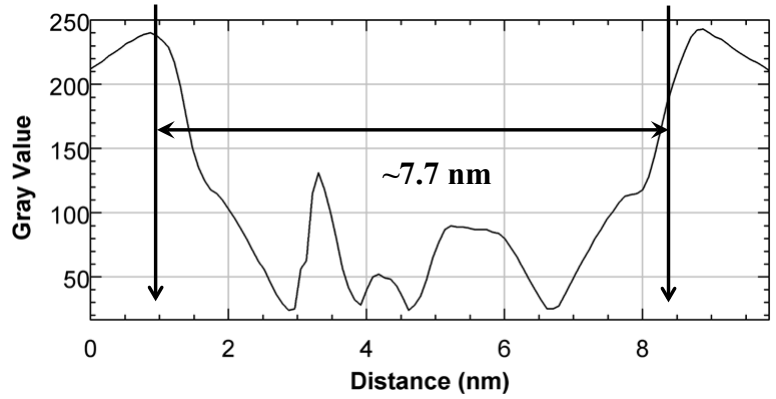

Figure 14: (a) A close-up the surface in Figure 11 (b) An analysis of the above graph along the yellow line, showing the size of a single QD is $\sim 7.7 \mathrm{~nm}$. A size distribution is visible, that may be quantified by determining the sizes for each particle.

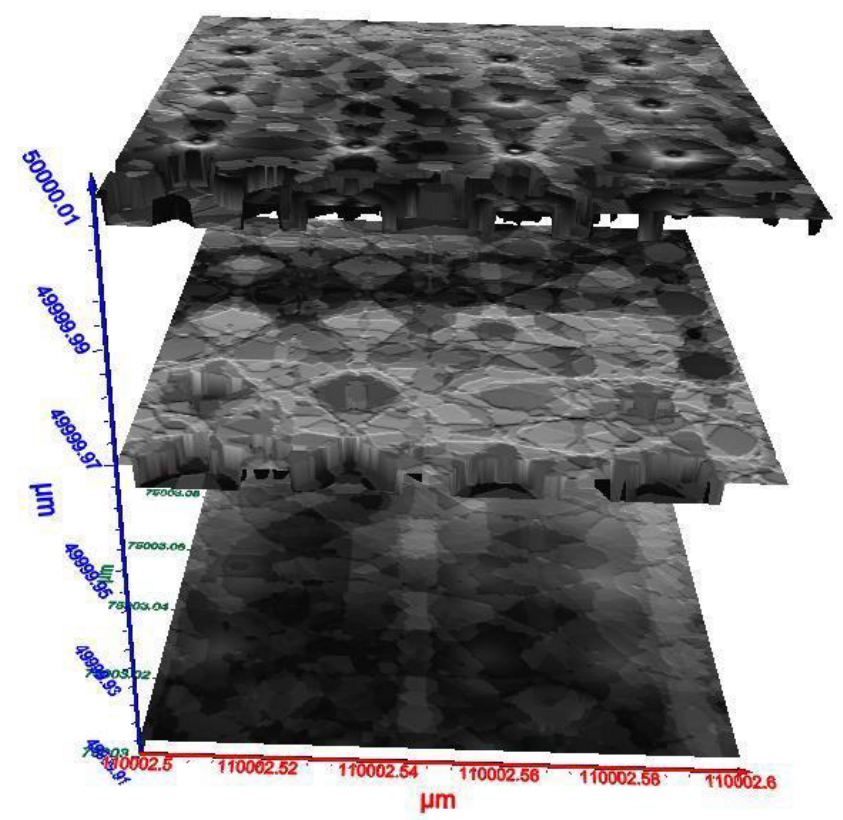

Figure 15: Three different slices extracted from the volume of Figure 10. This shows the ability for layer by layer inspection.
Figure $14 \mathrm{~b}$, the particle along the line of the image has a diameter of $\sim 7.7 \mathrm{~nm}$. Sizes of other particles and also that of the grains may be determined individually by similar procedure; from which the size distribution may be computed.

Thus, it has been demonstrated that the nanoscanner described in the present paper is capable of non-destructive and non-contact $3 \mathrm{D}$ imaging of AgI QDs. The PXRD and TEM characterization shows that the average size of the QDs is $(11.4 \pm 4.5) \mathrm{nm}$, while the terahertz image analysis reports the size of a representative single QD is $\sim 7.7$ $\mathrm{nm}$. This is within the range of the TEM analysis (Figure 9), thus is in agreement. The 3D image shows that most of the QDs are on the top layer of the film with very few under the top layer; however, there are no more particles along the thickness of the film. Further analysis could be carried out to establish the statistical variance of the size of the QDs and thereby a distribution with standard deviation could be estimated. Figure 15 exhibit three representative slices extracted from the volume of Figure 10 that was divided in to 100 slices. This demonstrates the ability for layer by layer inspection (Figures 9 to 15).

\section{Conclusions}

In this paper, reconstructive, $3 \mathrm{D}$, sub-surface imaging technique has been reported. The imaging is accomplished by means of terahertz nanoscanner in a non-contact and non-destructive fashion, with 
Citation: Rahman A, Rahman AK, Yamamoto T, Kitagawa H (2016) Terahertz Sub-Nanometer Sub-Surface Imaging of 2D Materials. J Biosens Bioelectron 7: 221. doi: 10.4172/2155-6210.1000221

Page 8 of 8

minimal sample preparation requirements. A dendrimer dipole excitation based CW terahertz source was used for the design of the nanoscanner. Gridding with inverse distance to power equations was utilized for reconstructive imaging. Both $2 \mathrm{D}$ and $3 \mathrm{D}$ images were utilized for unique identification of features and/or defects. Layer by layer inspection capability has been demonstrated. Graphical analysis was used for the QDs' size and layer thickness determinations. Nanometer resolution was achieved with accurate measurement of nanoparticles' size and layer thicknesses. AgI quantum dots have been measured for their size parameter. The size determined by the current terahertz imaging agrees within the results obtained via transmission electron microscope within the experimental error limits. It was also found that the techniques deployed here is effective for visualizing the 3D image of the samples. Thus, the reported non-contact measurement system may be deployed for characterizing $2 \mathrm{D}$ and $3 \mathrm{D}$ naomaterials as well as for process development and/or quality inspection at the production line.

\section{References}

1. Rahman A, Rahman AK (2012) Wide Range Broadband Terahertz Emission From High $X^{(2)}$ Dendrimer. In: Laurence $P$ (ed.) Terahertz Technology and Applications V, $82610 \mathrm{H}$.
2. Rahman A, Rahman AK, Tomalia DA (2016) Dendrimer Dipole Excitation: A New Mechanism for Terahertz Generation. J Biosens Bioelectron 7: 1

3. Rahman A, Rahman AK (2014) Effective Testing for Wafer Rejects Minimization by Terahertz Analysis and Sub-Surface Imaging. ASMC 2014, IEEE. pp. 151-155.

4. Davis JC (1986) Statistics and Data Analysis in Geology. John Wiley and Sons, New York.

5. Franke R (1982) Scattered Data Interpolation: Test of Some Methods. Mathematics of Computations 33: 181-200.

6. Auston DH, Glass AM, Ballman AA (1972) Optical Rectification by Impurities in Polar Crystals. Phys Rev Lett 28: 897.

7. Simon U, Miller CE, Bradley CC, Hulet RG, Curl RF, et al. (1993) DifferenceFrequency Generation in AgGaS2 by use of Single-Mode Diode-Laser Pump Sources. Optics Letters 18: 1062.

8. Rahman AK, Rahman A (2015) Terahertz Sub-Surface Scanning and 3D Imaging for Semiconductor Wafer Defect Detection. Pacifichem, Paper no. 955.

9. Ballroom A (2015) Marriott Waikiki Beach. Honolulu, HI.

10. Makiura R, Yonemura T, Yamada T, Yamauchi M, Ikeda R, et al. (2009) SizeControlled Stabilization of the Superionic Phase to Room Temperature in Polymer-Coated Agl Nanoparticles. Nat Mater 8: 476. 